\title{
Characterization of transverse anisotropy in Poisson's ratio using three-point radial deformation measurements
}

\author{
Nabi Kartal Toker ${ }^{1, *}$ and Kemal Sercan Büyükyılmaz ${ }^{2}$ \\ ${ }^{1}$ Middle East Technical University, Department of Civil Engineering, 06800 Ankara, Turkey \\ ${ }^{2}$ Yapı Merkezi, 34676 Istanbul, Turkey
}

\begin{abstract}
Poisson's ratio ( $v$ ) of rock specimens can be experimentally determined from radial deformation measurements in an unconfined compression test. This is commonly achieved by mounting a compressometer with diametric or perimetric measurement capability, both of which assume the specimen cross-section remains a circle. If there is anisotropy among the horizontal directions, this assumption fails and measurements err. In this study, a compressometer with three radial deformation measurements is developed. The accuracy of new compressometer in determining the mean of the two $v$ values along the two axes of anisotropy is theoretically investigated, assuming the deformed specimen is an ellipse in cross-section. Then, to capture separate $v$ values along the axes of anisotropy, data from actual tests employing the new device were analysed in two different approaches: Assuming the centroid of the cross-section remains stationary (1) does not produce physically reasonable results. Assuming the specimen moves laterally and $v$ values are constant (2) is possible through a grid search-type optimization algorithm. This results in calculation of the orientation of axes of anisotropy with respect to the compressometer, as well as the corresponding $v$ value.
\end{abstract}

\section{Introduction}

There are several methods in the literature to obtain Poisson's ratio value from a rock specimen. These traditional methods are conducted mainly by using Unconfined Compression Test (UCT) and with special apparatus to capture radial strains along with the axial deformation. Common ways to capture radial strain are directly recording circumferential or diameter displacements. If a compressometer frame is used, it is possible to employ a chain with an extensometer is wrapped around the perimeter at mid-height of the specimen [1] or clipping gauges for direct measurement of diameter changes [2]. Other methods (without the need for a compressometer) are strain gauges mounted at two or three points to record circumferential strains [3] or calculating radial deformations from digital image processing techniques applied on high-resolution, highspeed camera images of the specimen during tests [4]. All of these methods assume a unique Poisson's ratio for the rock specimen, and do not consider anisotropy of rock properties between the horizontal axes. Such traverse anisotropy can easily be present in rocks that have bedding planes or joints dominant in any alignment other than the horizontal. This results in two principal Poisson's ratio values $\left(v_{1}, v_{2}\right)$ along the axes of anisotropy.

A new method called "3-Point Radial Measurement", which uses a newly developed compressometer, is introduced in this study. Similar to other methods listed from literature, the proposed device is capable of obtaining a unique Poisson's ratio value. Moreover, anisotropic deformed shape and anisotropic Poisson's values also can be captured by the proposed measurement device when its data is processed to model the deformed cross section numerically.

\section{Reduction of anisotropic Poisson's ratios to a single value}

When ignoring anisotropy, conventional methods listed above are able to deliver a single Poisson's ratio by assuming the cross section remains circular. First, use of compressometer-based methods (including the proposed device) on a circular cross section that deforms into an ellipse is simulated through geometry calculations, to assess how the average $v$ they measure matches the actual average of the values along the axes of anisotropy.

\footnotetext{
* Corresponding author: toker@metu.edu.tr
} 


\subsection{Diametric measurement}

Direct diametric measurement is generally conducted by using adversely located displacement measurement points in classical Unconfined Compression Test (UCT). They are connected to a displacement measurement device, such as a Linear Potentiometer (LP). Before any loading, this LP reading corresponds to the initial diameter $\left(D_{0}\right)$ of the specimen. These two gauges convey the radial deformations of the specimen in opposite directions to the LP. Therefore, changes in the LP measurement while loading the specimen correspond to change in diameter $(\Delta D)$ of the specimen. Procedure of calculation Poisson's ratio of the specimen from the final diameter is as follows:

$$
v=\frac{\varepsilon_{r}}{\varepsilon_{z}}=\frac{\Delta D}{D_{0}}
$$

where $\varepsilon_{r}$ is the radial or diametric strain, $\varepsilon_{z}$ is the longitudinal or axial strain.

Poisson's ratio found with this apparatus is dependent on the utterly random initial location of gauges in relation to the anisotropy axes in the horizontal plane. This method therefore generates a random result between the principal Poisson's ratio values in the two horizontal axes of anisotropy, utterly failing to capture even an average Poisson's ratio if the specimen has transverse anisotropy.

\subsection{Perimetric measurement}

Perimetric measurement reaches a unique average value by recording the change in perimeter rather than change in diameter [5]. This method is generally conducted by using axial and circumferential LPs in classical UCT. A free-end chain is tightly wrapped around the perimeter of the specimen and these free-ends are attached to an LP to record the changes in the specimen perimeter. Therefore, before loading, LP reading corresponds to the initial perimeter, $\left(C_{i}\right)$ and during the experiment, the LP records deformation in terms of changes in perimeter, $(\Delta C)$. By calculating the change in perimeter, this method gives an average Poisson's ratio independent from the direction in a plane, so it also neglects the transverse anisotropy in rocks. Average Poisson's ratio calculations, which assume the cross-section remains a perfect circle after deformation, are as follows:

$$
\frac{\Delta C}{C_{i}}=\frac{\Delta r}{r_{0}}=\varepsilon_{r}
$$

where $r_{0}$ is the initial radius and $\Delta r$ is the change in the radius of the rock specimen.

The single $v$ value obtained from this method (using Ramanujan II numerical approximation for the perimeter of an ellipse [6]) is found to be equal to the arithmetic average of the principal Poisson's ratios, to the accuracy of 0.0004 when $v_{1}-v_{2}$ difference is as large as 0.5 .

\subsection{Three-point radial measurement}

Figure 1 illustrates the proposed compressometer frame. The device consists of 3 metal rings that surround the specimen, in order to assemble the gauges independently from specimen. It employs three LPs located at midheight, transversely $120^{\circ}$ apart from each other to record radial deformations. It has also two LPs, parallel to longitudinal axis, in order to record longitudinal deformations in UCT. These devices have resolution of $0.72 \mu \mathrm{m}$ with the 16-bit data acquisition system used in this setup. Height of the compressometer rings and therefore measurement point locations are adjustable, in order to accommodate different heights of specimens cut from $48 \mathrm{~mm}(\mathrm{NQ})$ and $63.5 \mathrm{~mm}(\mathrm{HQ})$ diameter core samples.

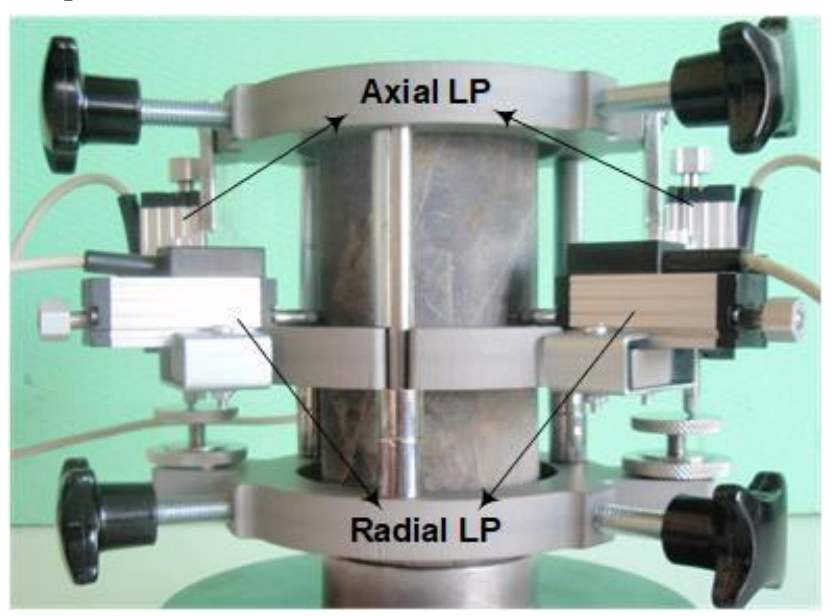

Fig.1. Picture of proposed compressometer. The third LP is behind the specimen.

An average Poisson's ratio can be calculated with this experiment, by finding average horizontal displacement $(\bar{\Delta})$, which is simply the arithmetic mean of radial displacement measurements of LP1 $\left(\Delta_{1}\right)$, LP2 $\left(\Delta_{2}\right)$, and LP3 $\left(\Delta_{3}\right)$, and calculating average Poisson's ratio value from the deformed shape's radius:

$$
v=\frac{\varepsilon_{r}}{\varepsilon_{z}}=\frac{2 \cdot \bar{\Delta}}{D_{0} \cdot \varepsilon_{z}}
$$

Figure 2 presents a deformed shape of a rock specimen together with axes of anisotropy, and three arbitrarily placed equidistant radial measurement points as the proposed measurement device has. Final deformed shape is assumed as an ellipse and horizontal deformation $(\Delta)$ in any direction can be written as a function of primary Poisson's ratio $\left(v_{1}\right)$, secondary Poisson's ratio $\left(v_{2}\right)$, initial radius $\left(r_{0}\right)$, longitudinal strain $\left(\varepsilon_{z}\right)$, and angle between positive $\mathrm{x}$-axis and LP1 $(\theta)$ through the following calculations. 


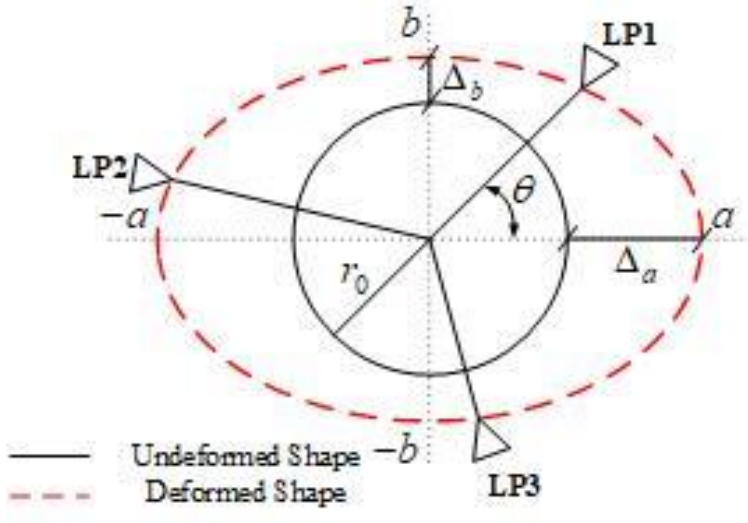

Fig.2. Undeformed and deformed shape for M3

The radial deformation measured by any LP at any point on the perimeter, expressed in terms of the Cartesian coordinates of the deformed shape, is:

$$
\Delta=\sqrt{x^{2}+y^{2}}-r_{0}
$$

Along the axes of anisotropy, radii of the ellipse are:

$$
\begin{aligned}
& a=r_{0}+\Delta_{a}=r_{0}+\varepsilon_{z} \cdot v_{1} \cdot r_{0} \\
& b=r_{0}+\Delta_{b}=r_{0}+\varepsilon_{z} \cdot v_{2} \cdot r_{0}
\end{aligned}
$$

Formulation of $\Delta$ can be derived with the help of general ellipse equation and trigonometry, as follows:

$$
\begin{aligned}
& \frac{x^{2}}{a^{2}}+\frac{y^{2}}{b^{2}}=1 \\
& y=x \cdot \tan \theta
\end{aligned}
$$

Therefore,

$$
\begin{gathered}
x=\sqrt{\frac{1}{\frac{1}{a^{2}}+\frac{\tan ^{2} \theta}{b^{2}}}} \\
y=\sqrt{\frac{1}{\frac{1}{a^{2}}+\frac{\tan ^{2} \theta}{b^{2}}} \cdot \tan \theta}
\end{gathered}
$$

Inserting equations 5 and 8 into the general equation 4 gives the following final expression of radial deformation as a function of $v_{1}, v_{2}, r_{0}, \varepsilon_{z}$ and $\theta$.

$$
\Delta=\frac{r_{0} \cdot \sec \theta}{\sqrt{\frac{1}{\left(1+\varepsilon_{z} \cdot v_{1}\right)^{2}}+\frac{\tan ^{2} \theta}{\left(1+\varepsilon_{z} \cdot v_{2}\right)^{2}}}}-r_{0}
$$

However, since there are three LPs that measure transverse deformation $120^{\circ}$ apart from each other, three different $\Delta$ values are measured at three different locations. Therefore, by taking average of these three $\Delta$ values using $\theta, \theta+120^{\circ}$ and $\theta+240^{\circ}$, where $\theta$ is a random angle between $0^{\circ}$ and $120^{\circ}$, one can obtain an average horizontal displacement $(\bar{\Delta})$ and use it to find a single average Poisson's ratio, by equation 3 .

In order to show the difference between traditional assumption of isotropy and possible range of anisotropic deformations, a parametric study is conducted. The radial displacement measurements are calculated for ellipse sections that are created by combinations of $v_{1}$ and $v_{2}$ values. The "average" Poisson's ratio values that are calculated from the average of three radial displacements are compared to the real arithmetic mean of $v_{1}$ and $v_{2}$.

In order to generate an example calculation, $r_{0}$ and $\varepsilon_{z}$ were chosen as $25 \mathrm{~mm}, 2.5 \%$, respectively. However, these have no effect on the result as all calculations are in the form of ratios. In these simulated scenarios, anisotropy in Poisson's ratio was generated by varying $v_{1}$ value from 0 to 0.5 with an increment of 0.1 and varying $v_{2}$ value from 0 to 0.5 with an increment of 0.01 . All calculations were conducted in a spreadsheet for all Poisson's ratio combinations. The angle $\theta$ between first LP and positive $\mathrm{x}$-axis was also used as a variable at first, but due to the periodic axisymmetry of the of the problem the largest change it could cause in average Poisson's ratio calculation is found to be a negligible $1.2 \times 10^{-5}$. The average Poisson's ratio values, which are calculated from equation 3 using theoretically simulated radial displacement readings around an ellipse by equation 9, were compared to the arithmetic mean of assumed $v_{1}$ and $v_{2}$ values, through an absolute error definition given by equation 10 . Figure 3 shows the results, which demonstrate miniscule errors in a parabolic relationship in equation 10 , to the difference between the principal Poisson's ratios:

$$
\text { Error }=\left|v-\frac{v_{1}+v_{2}}{2}\right|=0.004633\left(v_{1}-v_{2}\right)^{2}
$$

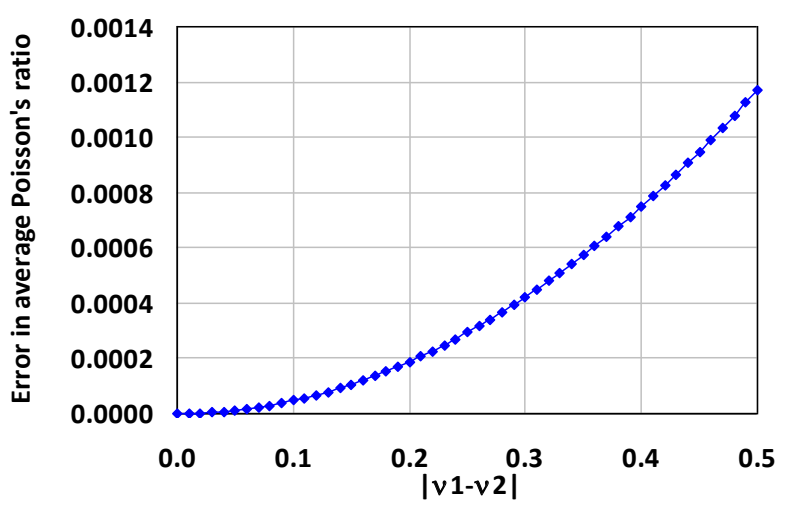

Fig.3. Error values of absolute difference of Poisson's ratios for 3-point radial measurement method.

\section{Numerical approaches for transverse anisotropy calculations}

Using the proposed compressometer device, the transverse anisotropy can be considered and principal Poisson's ratio values can be calculated by assuming that the specimen cross-section deforms into an ellipse. As 
three points are not enough to define a unique ellipse, an additional assumption is needed. Two different numerical approaches, each based on a different additional assumption, are presented in this part with their results. All calculations are carried out by using MATLAB computational software.

Unlike the previous part, the validation of applicability of these calculation approaches requires an unknown deformed shape. To provide it, real data from HQ-sized rock core specimens, which are equipped with the proposed device during UCT, are analyzed using both approaches. The strength and deformation properties of the rock specimens were selected to cover a wide range (Table 1 and Figure 4), from various core samples found in the lab. Length between the longitudinal measurement rings of the device is 107.5 $\mathrm{mm}$.

Table.1. Properties of tested rock core specimens.

\begin{tabular}{l|c|c|c|} 
& Test $\mathbf{1}$ & Test $\mathbf{2}$ & Test $\mathbf{3}$ \\
\hline $\mathrm{q}_{\mathrm{u}}(\mathrm{MPa})$ & 6.0 & 73 & 22 \\
$\mathrm{E}(\mathrm{MPa})$ & 850 & 66000 & 7600
\end{tabular}

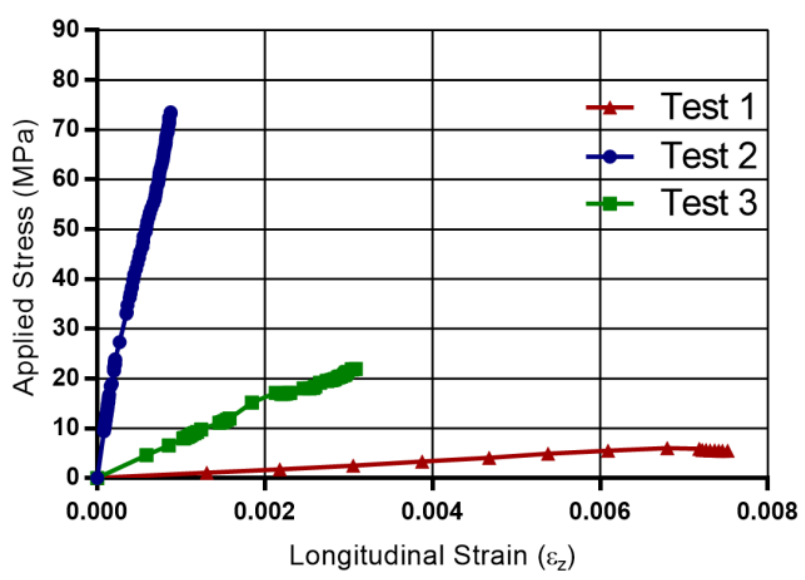

Fig.4. Stress - strain relationship obtained from the trial tests.

\subsection{Approach 1: Fixed centre, free to rotate}

In the simpler approach, the centre of the specimen cross-section is assumed to be stationary throughout the test. So, from three different transverse deformation readings obtained from LP1, LP2, and LP3 an ellipse has to be formed with exactly same centre coordinates with original shape.

At any moment or longitudinal strain during an experiment, the following data is measured: $\Delta_{i}$ (transverse deformation monitored by $i^{\text {th }}$ Linear Potentiometer, LP $i$, where $i$ can be 1, 2 or 3), $\Delta_{z 1}$ and $\Delta_{z 2}$ (longitudinal deformations recorded by vertical LPs), and applied force.

By referring to Figure 2, there are three unknowns: coordinates of point $(a)$ which represents primary axis of the ellipse and also serves as the x-axis in the calculations, coordinates of point $(b)$ which stands for the secondary axis of the ellipse and also serves as the yaxis in the calculations, and angle $(\theta)$ which is the angle between LP1 and positive x-axis.
Three equations can be obtained from transversely located LPs. First, transverse deformations should be converted to $\mathrm{x}$ and $\mathrm{y}$ coordinates by considering their corresponding angles with $\mathrm{x}$-axis. This transformation yields the coordinates of the final locations of measurement points.

$$
\begin{aligned}
& x_{i}=\left(r_{0}+\Delta_{i}\right) \cdot \cos \left(\theta+(i-1) \cdot 120^{\circ}\right) \\
& y_{i}=\left(r_{0}+\Delta_{i}\right) \cdot \sin \left(\theta+(i-1) \cdot 120^{\circ}\right)
\end{aligned}
$$

By inserting each point in to general ellipse equation (6), which the coordinates of the points must satisfy, three independent equations with three unknowns $(a, b$ and $\theta$ ) are obtained:

$$
\begin{aligned}
& {\left[\frac{\left(r_{0}+\Delta_{i}\right) \cdot \cos \left(\theta+(i-1) \cdot 120^{\circ}\right)}{a}\right]^{2}+} \\
& {\left[\frac{\left(r_{0}+\Delta_{i}\right) \cdot \sin \left(\theta+(i-1) \cdot 120^{\circ}\right)}{b}\right]^{2}=1}
\end{aligned}
$$

Newton-Jacobi method [7] is applied to every set of readings in the experiment, to solve for this non-linear equation system's unknowns, which are then related to the primary and secondary Poisson's ratios. For every reading step (denoted by index $t$ ) of the experiment, Newton-Jacobi procedure is repeated to converge to values of $a_{\text {final }}^{t}, b_{\text {final }}^{t}$, and $\theta_{\text {final. }}$. Longitudinal strain, $\varepsilon_{z}^{t}$, is calculated based on the initial specimen length and the average of two deformation readings measured by the vertical LPs.

Finally, one can easily use these parameters in equations 5, to obtain primary and secondary Poisson's ratios for each step as:

$$
v_{1}^{t}=\frac{a_{\text {final }}^{t}-r_{0}}{r_{0} \cdot \varepsilon_{z}^{t}} \quad \text { and } \quad v_{2}^{t}=\frac{b_{\text {final }}^{t}-r_{0}}{r_{0} \cdot \varepsilon_{z}^{t}}
$$

Figure 5 shows the change of primary and secondary Poisson's ratio $\left(v_{1}, v_{2}\right)$, and angle, $(\theta)$ all of which are calculated by the procedure described above with respect to longitudinal strain $\left(\varepsilon_{z}\right)$.

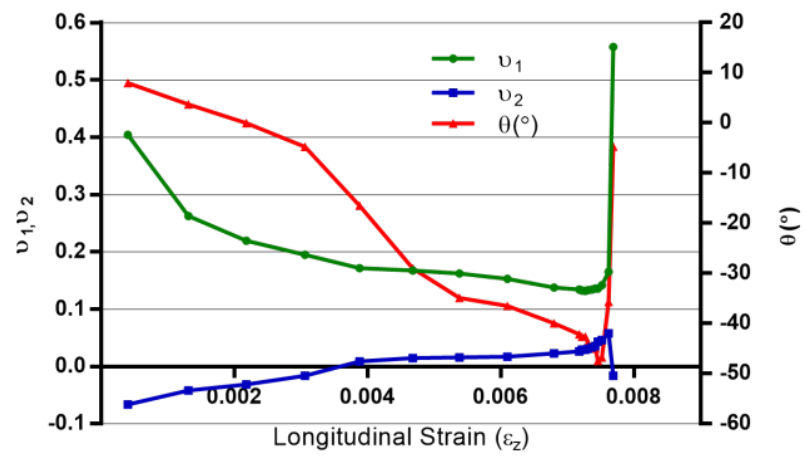

Fig.5. Variation of $v_{1}, v_{2}$ and $\theta$ with $\varepsilon_{z}$ from Test 1.

As seen in Figure 5, calculated values of primary and secondary Poisson's ratios show increasing or decreasing trend rather than staying around a constant value. Also, $(\theta)$ angle, between the primary axis of the ellipse and 
LP1 changes between $10^{\circ}$ and $-50^{\circ}$. Theoretically, angle between the primary axis of ellipse and LP1 should not change significantly since the horizontal enlargement of the specimen should align with natural axes of anisotropy. Minor angle changes can be accepted, since formation and propagation of new cracks can affect the anisotropy direction. Likewise, analyses of data from Tests 2 and 3 also result in variation of $\theta$ by 35 and $45^{\circ}$, respectively. These large changes in orientation of anisotropy calculated by this approach are attributed to the assumption that keeps the coordinates of centre constant throughout the deformation. In the light of these findings, it is concluded that assumptions based on the constant centre yield unrealistic responses; therefore, this assumption must be changed and horizontal movement of the cross section must be considered.

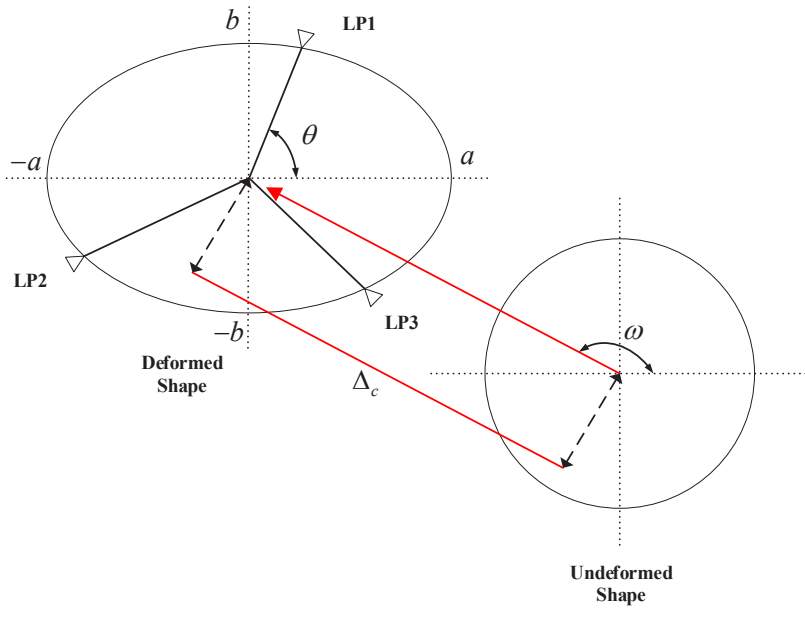

Fig.6. Deformed shape assumption of Approach 2 (displacement of the centre is vastly exaggerated).

\subsection{Approach 2: No rotation, centre moves linearly}

When centre of specimen is moving, movement direction must also be computed. Therefore, the unknowns are rotation angle between the primary axis and LP1 $(\theta)$, direction angle between direction of centre displacement and primary axis $(\omega)$, displacement of the centre $\left(\Delta_{C}\right)$, primary radius $(a)$, and secondary radius $(b)$ (Figure 6). This approach also assumes that primary and secondary Poisson's ratios are supposed to stay constant under increasing strains.

As in Approach 1, there are only 3 equations obtained by putting measurement points into the ellipse equation. However, this approach has 5 unknowns. In order to handle these unknowns a grid search algorithm [8] with $\theta$ and $\omega$ as two variables is conducted between $0-360^{\circ}$ with $0.1^{\circ}$ intervals. This leaves only 3 unknowns for each grid point, so Newton-Jacobi method is applied to all $\theta$ and $\omega$ combinations in order to find the remaining unknowns, $\Delta_{C}, a$, and $b$. This results in the ability to calculate principal Poisson's ratios $\left(v_{1}, v_{2}\right)$ at every strain of every angle combination. Root-Mean Square (RMS) Errors are calculated according to Poisson's ratio values averaged over the elastic strains of the test $\left(\overline{v_{1}}\right.$ and $\left.\overline{v_{2}}\right)$. Sum of these two RMS errors are taken as the total error value for that $\theta$ and $\omega$ combination. Finally, the $\theta$ and $\omega$ combination which gives the minimum total error in calculated Poisson's ratios from constant/average values are chosen as the final values of the angles. Corresponding average principal Poisson's ratios characterize the material's anisotropic behaviour, assuming they don't vary with strain over the elastic range.

For Test 1 , the angle combination which gives the minimum error is $\theta=26.0^{\circ}$ and $\omega=347.3^{\circ}$. Figure 7 shows for these angles, the principal Poisson's ratios and centre movement, $\Delta_{c}$, calculated at each longitudinal strain, $\varepsilon_{\mathrm{z}}$. The resulting $\bar{v}_{1}$ and $\bar{v}_{2}$ are 0.13 and 0.04 , respectively.

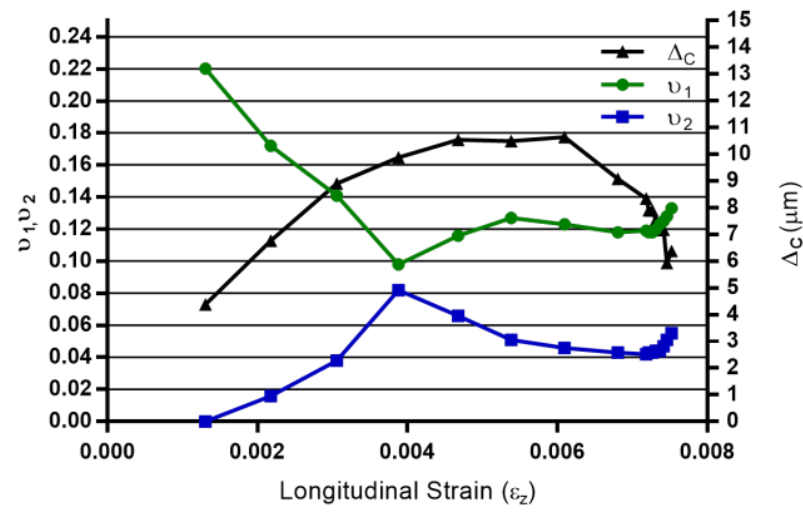

Fig.7. Variation of $v_{1}, v_{2}$ and $\Delta_{C}$ with longitudinal strain for Test1, as calculated by Approach 2.

The proposed numerical approach is capable of detecting the little anisotropy in this soft rock specimen with a relatively small Poisson's effect. Result in Figure 7 indicates that centre of the specimen moves as the test progresses, and values of Poisson's ratios are strictly different from those obtained in Approach 1. Poisson's ratios obtained by Approach 2 show changes with strain as those by Approach 1 did, but for Approach 2, changes are smaller.

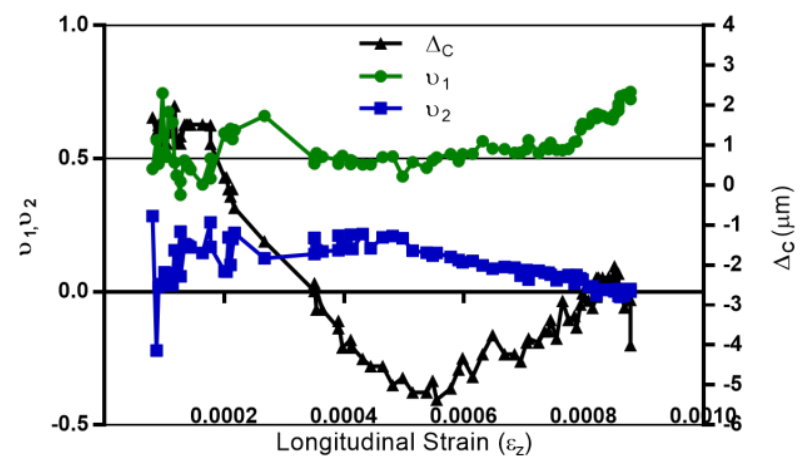

Fig.8. Variation of $v_{1}, v_{2}$ and $\Delta_{C}$ with longitudinal strain for Test2, as calculated by Approach 2. 


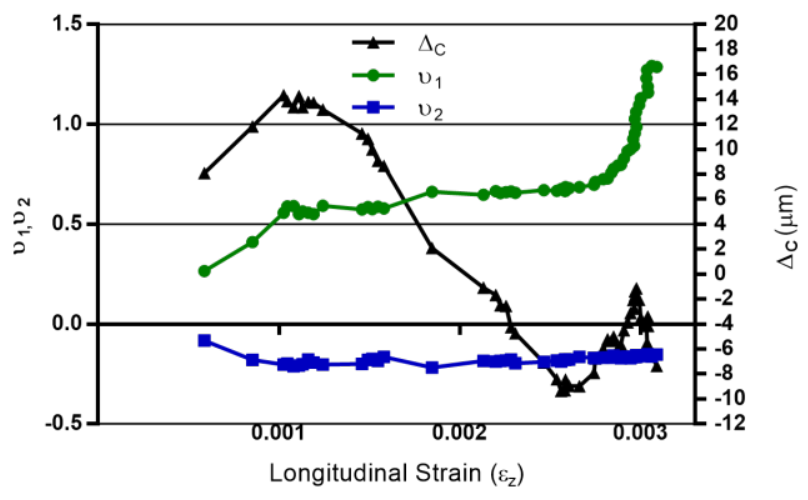

Fig.9. Variation of $v_{1}, v_{2}$ and $\Delta_{C}$ with longitudinal strain for Test3, as calculated by Approach 2.

The data from the other tests were also processed through the same algorithm. The results are presented in Figures 8 and 9, and summarized in Table 2.

Table.2. Summary of calculated angles and resulting Poisson's ratio values by Approach 2 .

\begin{tabular}{c|c|c|c|} 
& Test 1 & Test 2 & Test 3 \\
\hline$\theta\left(^{\circ}\right)$ & 26.0 & 48.4 & 32.8 \\
$\omega\left(^{\circ}\right)$ & 347.3 & 235.3 & 188.5 \\
\hline$\overline{v_{1}}$ & 0.13 & 0.55 & $0.77 *$ \\
$\overline{v_{2}}$ & 0.04 & 0.10 & -0.17 \\
\hline
\end{tabular}

Note that the results are affected by the deformation range of the test over which the algorithm is applied. In Test 3, one of the Poisson's ratios is found as a very high 0.77 , due to the onset of crack propagation towards the end of the test. If the aim is to model only the elastic range, one should limit the data fed into the algorithm (to less than 0.0028 axial strain in this case, which would result in $\overline{v_{1}}$ of 0.6 ).

\section{Conclusion}

A compressometer with three radial deformation measurement points is developed, along with a numerical algorithm that is able to extract the Poisson's ratio values along the axes of anisotropy.

In a simpler analysis, radial displacements measured by the device can be averaged to obtain an approximation to the arithmetic mean of the principal Poisson's ratios, just like other devices in practice can.

Moreover, 3-point radial measurement results can also be processed through a numerical interpretation scheme that deliver the two principal Poisson's ratio values. In order to obtain those values, two approaches are tested. Two numerical approaches were tried on actual test data. Approach 1, which assumes constant centre location, fails modelling the behaviour realistically by implying the axes of anisotropy are able to rotate by up to $60^{\circ}$. Approach 2, which assumes no rotation and searches for constant principal Poisson's ratios, appears to succeed in capturing the anisotropy by delivering Poisson's ratio values that are more stable over the course of an unconfined compression test.
Poisson's ratio values still show variation with strain, especially when getting out of the elastic range. However, the method can be used for the elastic range of the behaviour to converge to unique values of principal Poison's ratios.

We thank TOKER Drilling \& Construction Co. for providing test data and equipment for the study.

\section{References}

1. A. Kesonen, Mechanical properties of Finnish rocks based on uniaxial compressive strength and tensile strength test, AALTO University (2015)

2. K. N. Kaklis, S. P. Mavrigiannakis, Frattura ed Integrità Strutturale, 40, 18 (2017)

3. L. Suwal, R. Kuwano, Institute of Industrial Science, University of Tokyo, 45 (2012)

4. S. E. Salazar, Theses and Dissertations, (University of Arkansas), 2563, (2017)

5. L. Jacobsson, SKB P 06, 69 (2007)

6. S. Ramanujan, Ramanujan's Collected Works, (1962)

7. A. B. Israel, J. Math Anal. and Applic. 15, 243 (1966)

8. C-W. Hsu, C-C. Chang, C-J. Lin, A practical guide to support vector classification, National Taiwan University (2010). 